\title{
ARTICLE \\ Does relapse contribute to treatment resistance? Antipsychotic response in first- vs. second-episode schizophrenia
}

\author{
Hiroyoshi Takeuchi ${ }^{1,2}$, Cynthia Siu ${ }^{3}$, Gary Remington ${ }^{2,4,5,6}$, Gagan Fervaha ${ }^{2,7}$, Robert B. Zipursky ${ }^{2,4}$, George Foussias ${ }^{2,4,5,6}$ and $^{2}$ \\ Ofer Agid A $^{2,4}$
}

\begin{abstract}
Although some studies have suggested that relapse may be associated with antipsychotic treatment resistance in schizophrenia, the number and quality of studies is limited. The current analysis included patients with a diagnosis of first-episode schizophrenia or schizoaffective disorder who met the following criteria: (1) referral to the First-Episode Psychosis Program between 2003 and 2013; (2) treatment with an oral second-generation antipsychotic according to a standardized treatment algorithm; (3) positive symptom remission; (4) subsequent relapse (i.e., second episode) in association with non-adherence; and (5) reintroduction of antipsychotic treatment with the same agent used to achieve response in the first episode. The following outcomes were used as an index of antipsychotic treatment response: changes in the brief psychiatric rating scale (BPRS) total and positive symptom scores and number of patients who achieved positive symptom remission and 20 and $50 \%$ response. A total of 130 patients were included in the analyses. Although all patients took the same antipsychotic in both episodes, there were significant episode-by-time interactions for all outcomes of antipsychotic treatment response over 1 year in favor of the first episode compared to the second episode ( $50 \%$ response rate: 48.7 vs. $10.4 \%$ at week $7 ; 88.2$ vs. $27.8 \%$ at week 27 , respectively). Although antipsychotic doses in the second episode were significantly higher than those in the first episode, results remained unchanged after adjusting for antipsychotic dose. The present findings suggest that antipsychotic treatment response is reduced or delayed in the face of relapse following effective treatment of the first episode of schizophrenia.
\end{abstract}

Neuropsychopharmacology (2019) 44:1036-1042; https://doi.org/10.1038/s41386-018-0278-3

\section{INTRODUCTION}

Relapse is associated with considerable psychological and social distress in patients with schizophrenia and their families, as well as increased economic burden [1]. Continuous antipsychotic treatment plays a key role in preventing relapse in schizophrenia [2]; however, medication non-adherence occurs frequently [3], in particular early in the illness' course [4], resulting in increased risk of relapse [5].

The implications of relapse are not entirely clear. It has been established that antipsychotic treatment response is greater in first-episode schizophrenia [6, 7], and optimal antipsychotic doses are lower than later in the illness' course [8]. This has led to speculation that subsequent psychotic episodes (i.e., relapse) may be involved in the process of diminished antipsychotic treatment response (i.e., treatment resistance) [9]. In a recent review of this topic [10], it was noted that a small number of studies indicated that relapse was associated with treatment resistance. For example, Lieberman et al. treated 70 patients with first-episode psychosis, most antipsychotic naive, and followed them for 5 years [11]. Twenty-two patients remitted but subsequently relapsed (i.e., second episode), with six relapsing twice (i.e., third episode); all were treated with the same antipsychotic to which they responded. There were significant differences in median times to remission between the first and second episodes (8.4 vs. 11.9 weeks), as well as the second and third episodes (7 vs. 24.1 weeks). More recently, Emsley et al. evaluated 31 patients with first-episode psychosis who achieved response (50\% reduction in overall symptom severity) to long-acting injectable risperidone and, thereafter, received intermittent treatment for 3 years. Among those who experienced relapse (i.e., second episode), 5 patients (16.1\%) did not meet the same criteria for response to the same medication [12]. In a somewhat similar study, Emsley et al. reported on 97 patients with schizophrenia who responded to long-acting injectable paliperidone, thereafter, received placebo treatment for 1 year and experienced relapse during this period. Fourteen patients (14.4\%) failed to respond to the same medication after the relapse [13]. Taken together, these studies have suggested that relapse may be associated with antipsychotic treatment resistance in schizophrenia; however, to date the number and quality of studies are limited.

Further evidence arising from clearly defined treatment algorithms would prove valuable in advancing this line of investigation. To this end, we report on longitudinal follow-up data of first-episode patients with schizophrenia who were treated from the outset within such a framework, comparing trajectories of antipsychotic treatment response before and after relapse

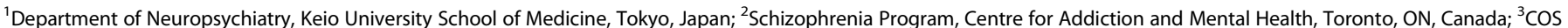

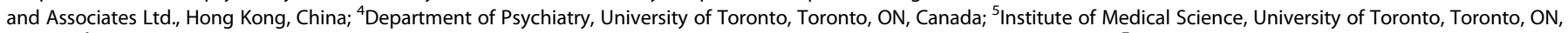

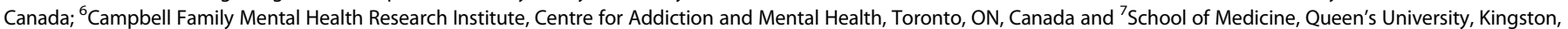
ON, Canada

Correspondence: Ofer Agid (Ofer.Agid@camh.ca)

Received: 10 July 2018 Accepted: 13 November 2018

Published online: 22 November 2018 
following initial response. We hypothesized that antipsychotic response would diminish in the second episode compared to the first episode.

\section{MATERIALS AND METHODS}

Patients

Patients included in the study were referred to the First-Episode Psychosis Program at the Centre for Addiction and Mental Health (CAMH), Toronto, Ontario, Canada, between 2003 and 2013, and followed-up by a community-based treatment team for firstepisode schizophrenia. A treatment algorithm standardizing pharmacologic management was implemented, in keeping with existing guidelines. Briefly, patients were offered trials with 2 oral second-generation antipsychotics, each in 3 stages with a maximum duration of 4 weeks as required and tolerated: Stage 1 -a low dose treatment (risperidone 2-3 mg/day, olanzapine 5-10 $\mathrm{mg} /$ day, quetiapine $300-400 \mathrm{mg} /$ day, aripiprazole $10-15 \mathrm{mg} /$ day, paliperidone 3-6 mg/day, and ziprasidone 40-120 mg/day); Stage 2-a full dose treatment (risperidone $4-6 \mathrm{mg} /$ day, olanzapine $12.5-20 \mathrm{mg} /$ day, quetiapine $425-800 \mathrm{mg} /$ day, aripiprazole $20 \mathrm{mg} /$ day, paliperidone $9 \mathrm{mg} /$ day, and ziprasidone $140-160 \mathrm{mg} /$ day); and Stage 3-a high dose treatment (risperidone $6.5-10 \mathrm{mg} /$ day, olanzapine $22.5-30 \mathrm{mg} /$ day, quetiapine $850-1200 \mathrm{mg} /$ day, aripiprazole $30 \mathrm{mg} /$ day, paliperidone $12 \mathrm{mg} /$ day, and ziprasidone 180-200 mg/day). Thereafter, clozapine was offered to those who continued to show suboptimal clinical response. The algorithm does not recommend the option of being without antipsychotic treatment during 5 years after the first episode. For patients who experienced relapse due to non-adherence (i.e., the second episode), the algorithm recommends the same antipsychotic and dose used to achieve response in the first episode following a short titration schedule (i.e., 2-3 days, with the opportunity to titrate further once the previous dose is achieved). Patients were advised that their treatment would be applied according to this algorithm, but that it would be flexibly administered in accordance with the individual-specific clinical condition and preferences. If a patient met substance abuse/dependence other than nicotine according to the DSM-IV, the patient was not followed-up with the algorithm.

To be included in the study, patients met the following criteria: (1) treatment for the first episode of psychosis was successful with an oral second-generation antipsychotic and resulted in meeting positive symptom remission criteria (see below); and (2) a second episode of psychosis (i.e., relapse [see below]) was experienced due to non-adherence.

Data for this study were collected in the course of routine clinical care and analyzed retrospectively. The Research Ethics Board at the CAMH approved this study and the publication of anonymized clinical data.

\section{Assessments}

In addition to collecting general demographic, clinical, and pharmacological information, the Diagnostic and Statistical Manual of Mental Disorders, Fourth Edition (DSM-IV) [14] diagnosis was made through clinical interview by a staff psychiatrist (O.A.) who oversaw the care and assessment of this cohort during treatment. All patients met criteria for schizophrenia or schizoaffective disorder, and for the purpose of reporting are referred to collectively as first-episode schizophrenia. Clinical ratings included the clinical global impressions scale (CGI) [15] and the 18-item brief psychiatric rating scale (BPRS) [16]. Positive symptom remission was defined as (a) the CGI-I score $\leq 2$ and (b) the BPRS 4 core psychotic items (conceptual disorganization; hallucinatory behavior; suspiciousness; unusual thought content) scores $\leq 3$. Relapse was defined as a $20 \%$ increase in the BPRS total scores at a regularly scheduled visit following positive symptom remission. Positive symptom relapse was defined as $\geq 4$ on at least 1 item of the BPRS 4 core psychotic symptoms.
Medication adherence was assessed through multiple measures, including patient and caregiver feedback, pharmacy reports regarding prescription utilization, and random pill counts and urine screens. For the data presented here, evidence from pill counts indicated an adherence rate of $\geq 80 \%$ unless otherwise stated.

\section{Data analysis}

The primary analysis outcome was changes in the BPRS total scores from individual baseline visit (prior to treatment for the first or second episode). Secondary analysis outcomes were changes in the BPRS positive symptom scores and the rates of patients achieving positive symptom remission. The proportions of patients achieving $\geq 20 \%$ and $\geq 50 \%$ reduction in the BPRS total scores (20\% responder and 50\% responder, respectively) were analyzed as additional outcome measures. Percent score changes were calculated using the following formulation [17, 18]: (BPRS total score at a given time-BPRS total score at baseline)/(BPRS total score at baseline-18) $\times 100$. We chose 20 and $50 \%$ as a threshold of response because these correspond to "minimal improvement" and "much improvement", respectively [19].

Mixed model for repeated-measures (MMRM) analysis was applied to compare mean change in BPRS score between the first and second episode of schizophrenia, which included fixed effects for episode, visit, and episode-by-visit interaction terms. An unstructured covariance matrix was used for within-patient correlation. In the analyses of remission and response rates for the first and second episodes, we applied a generalized estimating equation (GEE) model for longitudinal data that included terms for episode, visit, and episode-by-visit interaction effect. To account for antipsychotic doses, olanzapine equivalent antipsychotic doses were calculated according to the minimum effective dose method [20] and were added to the MMRM and GEE models as a covariate. In addition, logistic regression analyses were performed to examine the impact of demographic and clinical characteristics of patients including duration of unfollow-up between the first and second episodes (i.e., duration from the last visit of the first episode to the first visit of second episode) on patients who sustained positive symptom remission for 8 weeks or longer in the second episode vs. those who did not.

A 2-tailed $P$-value of $<0.05$ was considered statistically significant for all tests. All statistical analyses were conducted using the SAS 7.13 (SAS Institute Inc., Cary, NC, USA).

\section{RESULTS}

Demographic and clinical characteristics of patients

A total of 130 patients were included in the analyses. All patients were treated as outpatients, although 78 patients (60.0\%) and 67 patients $(51.5 \%)$ needed a short hospitalization during the first and second episode, respectively. Table 1 displays demographic and clinical characteristics of the patients. The patients visited the clinic for the first time at a mean \pm SD age of $22.1 \pm 3.1$ years and were followed for $33.5 \pm 19.7$ weeks. They stopped visiting the clinic for $19.3 \pm 9.7$ weeks, relapsed, and were followed-up again for $30.5 \pm 14.0$ weeks. No significant difference in duration of unfollow-up between the first and second episodes were found between the patients who sustained positive symptom remission in the second episode $(N=63)$ and those who did not $(N=67)$ $\left(19.7 \pm 7.3\right.$ weeks vs. $19.0 \pm 9.7$ weeks, respectively; $\mathrm{OR}=1.008, X^{2}$ $=0.17, P=0.68$ ) (Supplemental Table 1). At the first visit in the second episode, 126 of 129 patients (97.6\%) also met positive symptom relapse criteria. Psychopathology and treatment at first and second episodes are shown in Table 2. There was no significant difference in baseline BPRS total scores between the two episodes ( $59.1 \pm 5.9$ vs. $58.7 \pm 6.7 ; t=0.43, P=0.67)$. In the majority of patients risperidone or olanzapine was administered for the first episode, with the mean \pm SD dose of risperidone or 
olanzapine, $4.2 \pm 1.1$ or $15.5 \pm 6.2 \mathrm{mg} /$ day, respectively. All patients took the same antipsychotic at the second episode, although with the second episode doses ended up significantly higher than those in the first episode $(17.3 \pm 6.4 \mathrm{mg} /$ day vs. $16.6 \pm 7.4 \mathrm{mg} /$ day, respectively; $t=-2.19, P=0.03)$.

Changes in BPRS total and positive symptom scores over time in first vs. second episodes

Figure 1 illustrates changes in the BPRS total and positive symptom scores over time in the first and second episodes. There were significant episode-by-time interactions for both total and positive symptom trajectories over 1 year in the two episodes $(F=$ 13.8, $\mathrm{df}=15,2242, P<0.001 ; F=20.5, \mathrm{df}=15,2288, P<0.0001$, respectively), indicating that general and core symptom improvements were more rapid and greater in the first episode than the second episode. The results remained unchanged after adjusting

\begin{tabular}{|ll|}
\hline Table 1. Demographic and clinical characteristics of patients $(N=130)$ \\
\hline & $N(\%)$ or Mean \\
& $(\mathrm{SD})$ \\
\hline Male & $113(86.9)$ \\
Age at first episode (years) & $22.1(3.1)$ \\
Education & \\
Less than high school & $16(12.3)$ \\
Some high school & $50(38.5)$ \\
Completed high school & $38(29.2)$ \\
Some post-secondary education & $23(17.7)$ \\
Completed post-secondary education & $3(2.3)$ \\
Ethnicity & \\
Caucasian & $88(67.7)$ \\
Asian & $22(16.9)$ \\
African Canadian & $20(15.4)$ \\
Marital status & \\
Single & $125(96.2)$ \\
Married/long-term partnership & $5(3.8)$ \\
Diagnosis & $126(96.9)$ \\
Schizophrenia & $4(3.1)$ \\
Schizoaffective disorder & $19.3(9.7)$ \\
Duration of unfollow-up between first and second \\
episodes (weeks)
\end{tabular}

for antipsychotic doses $(F=13.8, \mathrm{df}=15,2241, P<0.001 ; F=20.5$, $\mathrm{df}=15,2287, P<0.0001$, respectively).

Changes in positive symptom remission and response rates over time in first vs. second episodes

Significant interactions between episode and time were also found for positive symptom remission rates (Fig. 2$)\left(X^{2}=95.0, P<\right.$ $0.001)$ and 20 and $50 \%$ response rates $\left(X^{2}=75.2, P<0.001 ; X^{2}=\right.$ 105.0, $P<0.001$, respectively) (Fig. 3); the results remained unchanged after adjusting for antipsychotic doses $\left(X^{2}=93.6, P\right.$ $<0.001 ; X^{2}=70.2, P<0.001 ; X^{2}=105.1, P<0.001$, respectively). As depicted in Figs. 2,3 , the majority of patients achieved positive symptom remission and $20 \%$ response in both the first and second episodes, with a faster attainment of this during the first episode; in contrast, more than half of patients failed to achieve $50 \%$ response in the second episode.

\section{DISCUSSION}

Our findings add to the limited body of evidence presently available linking relapse in schizophrenia with increasing treatment resistance. The uniqueness and strength of the present study are threefold. First, all data were collected as part of routine clinical practice, allowing results to be generalized to 'real world' clinical practice. Second, at the same time a clearly defined treatment algorithm permits data to be grouped and compared more systematically. Third, there was homogeneity in terms of the study population; specifically, all individuals had achieved positive symptom remission in response to a first antipsychotic trial, chose to discontinue treatment and relapsed, at which point they were reinstated on the same antipsychotic. The analyses demonstrated that antipsychotic treatment response was reduced and/or delayed in the second episode. In addition, higher doses ultimately prescribed in the second episode may be seen as evidence suggesting increased treatment resistance, mirroring other evidence indicating lower antipsychotic doses are needed in first-episode schizophrenia compared to multiple-episode schizophrenia [8]; however, antipsychotic dose did not have a significant impact on the difference in antipsychotic treatment response between the two episodes, indicating no clinical significance.

Recent studies have shown that there are 2 distinct types of treatment resistance [21-23]: the majority of patients with treatment-resistant schizophrenia present "early resistance" or "treatment resistance from the illness onset" not "late resistance" or "delayed-onset treatment resistance". As only patients who responded to an antipsychotic and achieved positive symptom

Table 2. Psychopathology and treatment at first and second episodes

\begin{tabular}{llll}
\hline & $\begin{array}{l}\text { First episode }(N=130) \\
N(\%) \text { or Mean (SD) }\end{array}$ & $\begin{array}{l}\text { Second episode }(N=130) \\
N(\%) \text { or Mean (SD) }\end{array}$ & $\begin{array}{l}\text { Between-episode difference } \\
P \text {-value }\end{array}$ \\
\hline Baseline BPRS total score & $59.1(5.9)$ & $58.7(6.7)$ & 0.51 \\
Baseline BPRS positive symptom score & $19.3(2.4)$ & $19.1(3.1)$ & 0.28 \\
Duration of follow-up (weeks) & $33.5(19.7)$ & $30.5(14.0)$ & 0.10 \\
Type of antipsychotics [highest dose of antipsychotics (mg/day)] & & & $0.66^{*}$ \\
Aripiprazole & $10(7.7)[16.4(5.1)]$ & $10(7.7)[17.0(4.2)]$ & $0.049^{*}$ \\
Olanzapine & $55(42.3)[15.5(6.2)]$ & $55(42.3)[16.5(4.4)]$ & $0.37^{*}$ \\
Paliperidone & $5(3.8)[6.6(2.5)]$ & $5(3.8)[7.2(2.7)]$ & $1.00^{*}$ \\
Quetiapine & $7(5.4)[743(190)]$ & $7(5.4)[743(98)]$ & $0.26^{*}$ \\
Risperidone & $53(40.8)[4.2(1.1)]$ & $53(40.8)[4.4(1.0)]$ & $17.3(6.4)$ \\
Olanzapine-equivalent highest dose of antipsychotics (mg/day) & $16.6(7.4)$ & & $\mathbf{0 . 0 3}$ \\
\hline BPRS bief & &
\end{tabular}


a

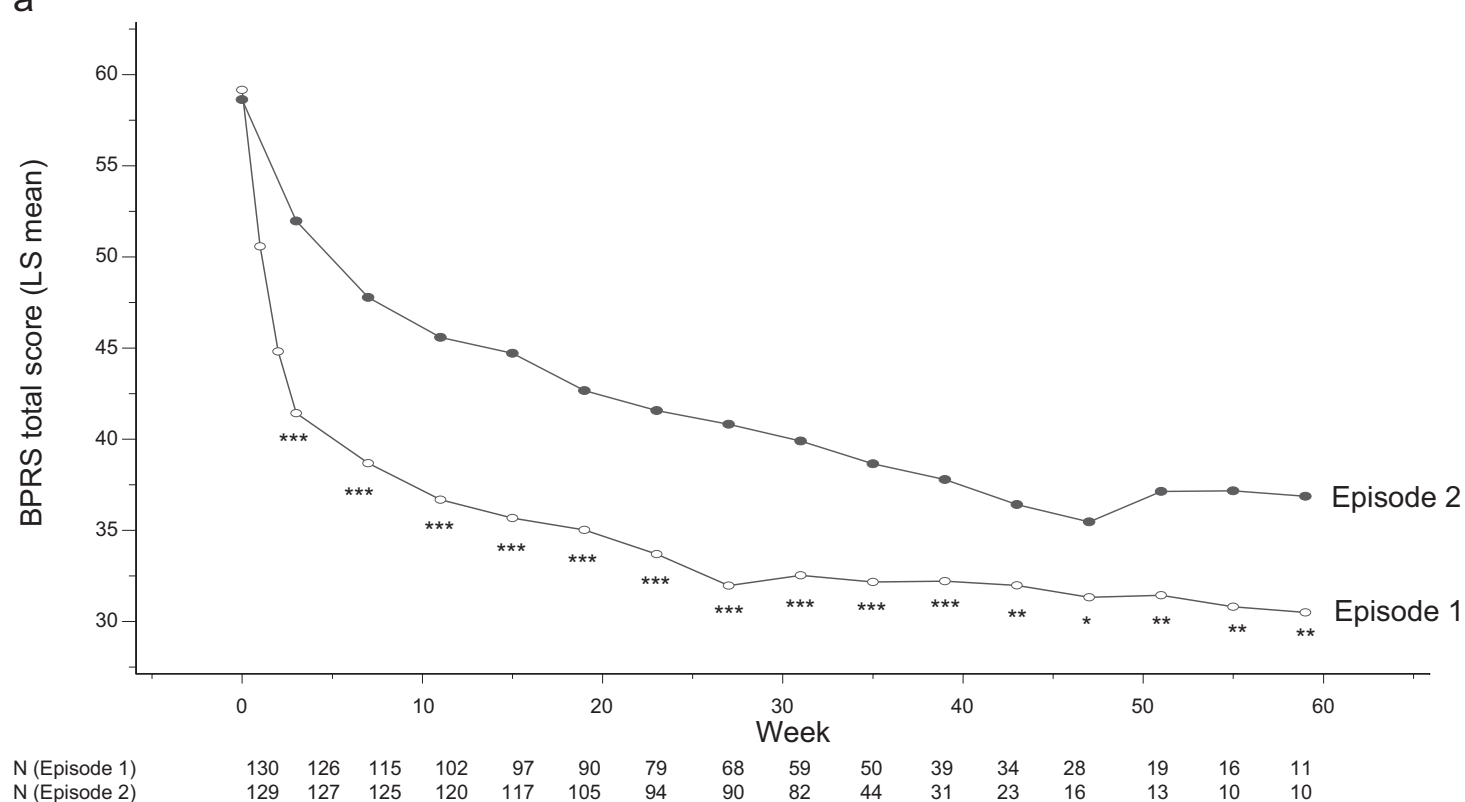

b

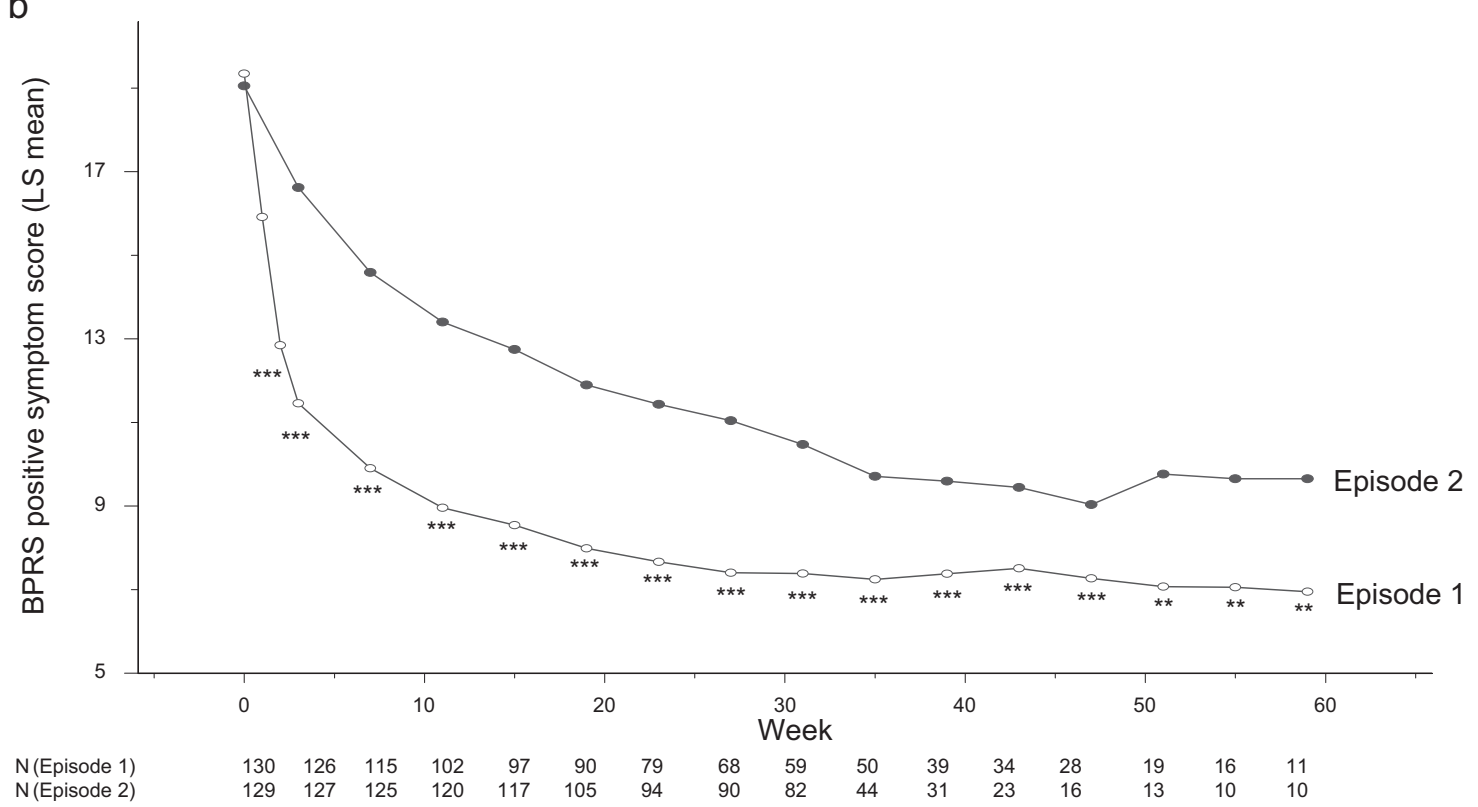

Fig. 1 a Changes in BPRS total scores over time in first vs. second episodes $(N=130)$. The mixed-model analysis revealed a significant interaction between episode and time $(F=13.8, \mathrm{df}=15,2242, P<0.001)$. ${ }^{* * *} P<0.001,{ }^{* *} P<0.01,{ }^{*} P<0.05$. BPRS brief psychiatric rating scale. b Changes in BPRS positive symptom scores over time in first vs. second episodes $(N=130)$. The mixed-model analysis revealed a significant interaction between episode and time $(F=20.5, \mathrm{df}=15,2288, P<0.0001)$. ${ }^{* *} P<0.001,{ }^{* *} P<0.01,{ }^{*} P<0.05$. BPRS brief psychiatric rating scale

remission were included in this analysis (i.e., the former population were excluded), the findings shed light on how treatment resistance evolves in schizophrenia. This strategy represents an important link in understanding those individuals with the latter form of treatment resistance as the distinction is lost at that point where both groups are assimilated under the term "treatmentresistant schizophrenia" [24]. From a mechanistic standpoint, it will be critical to evaluate each separately as it would seem that the underlying pathophysiology may well be different.

The present findings also emphasize the importance of relapse prevention in schizophrenia. The recent 2 reviews, in which experts on clinical psychopharmacology carefully scruitized the current evidence on long-term consequencies of antipsychotic treatment, have generally recommended long-term antipsychotic treatment in schizophrenia, because its benefits are weighed against its risks $[25,26]$. The present treatment algorithm engaged numerous strategies to encourage adherence, defined as $\geq 80 \%$ here, including pharmacy contacts, reports from patient/caregivers, and random pill counts as well as urine screens during the follow-up period. Despite this, a substantial number of patients discontinued treatment and relapsed. To this point, recent metaanalyses have shown superiority of long-acting injectable antipsychotics over oral antipsychotics for study discontinuation due to any reason as well as replase/hospitalization not in randomized controlled trials $[27,28]$, but in mirror-image studies [29] and cohort studies [30] which are closer to the real-world 
$\mathrm{H}$ Takeuchi et al.

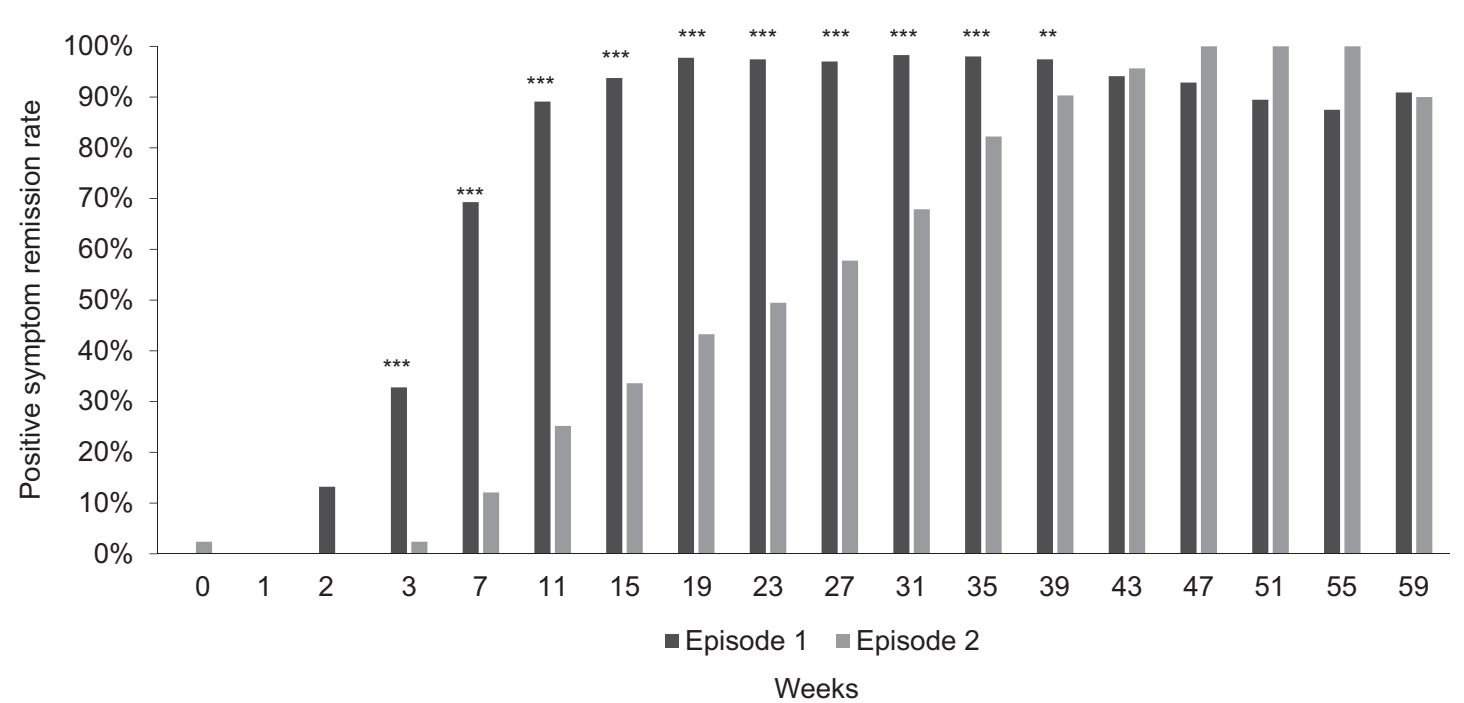

Fig. 2 Changes in positive symptom remission rates over time in first vs. second episodes $(N=130)$. The generalized estimating equation analysis revealed a significant interaction between episode and time $(F=95.0, P<0.001)$. ${ }^{* * *} P<0.001,{ }^{* *} P<0.01$

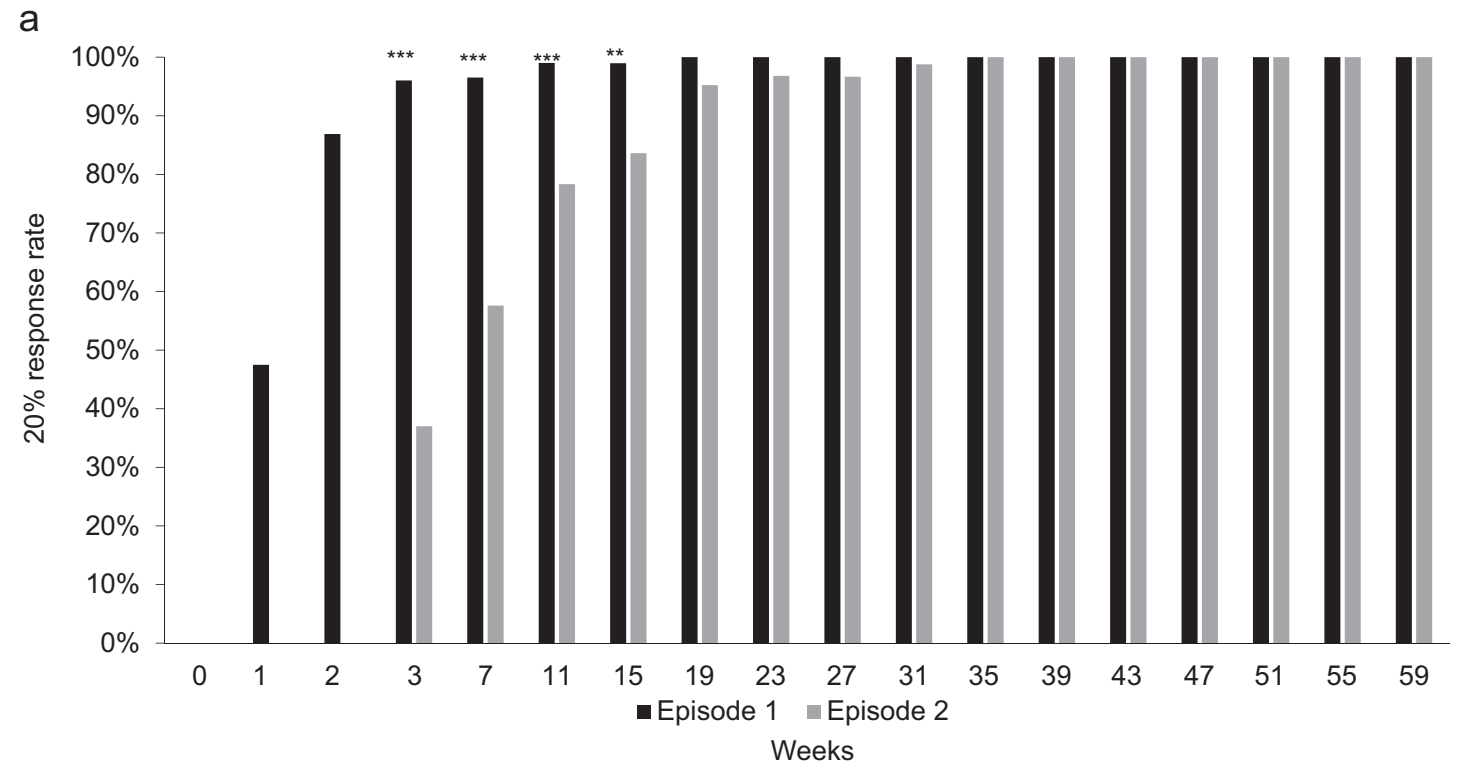

b

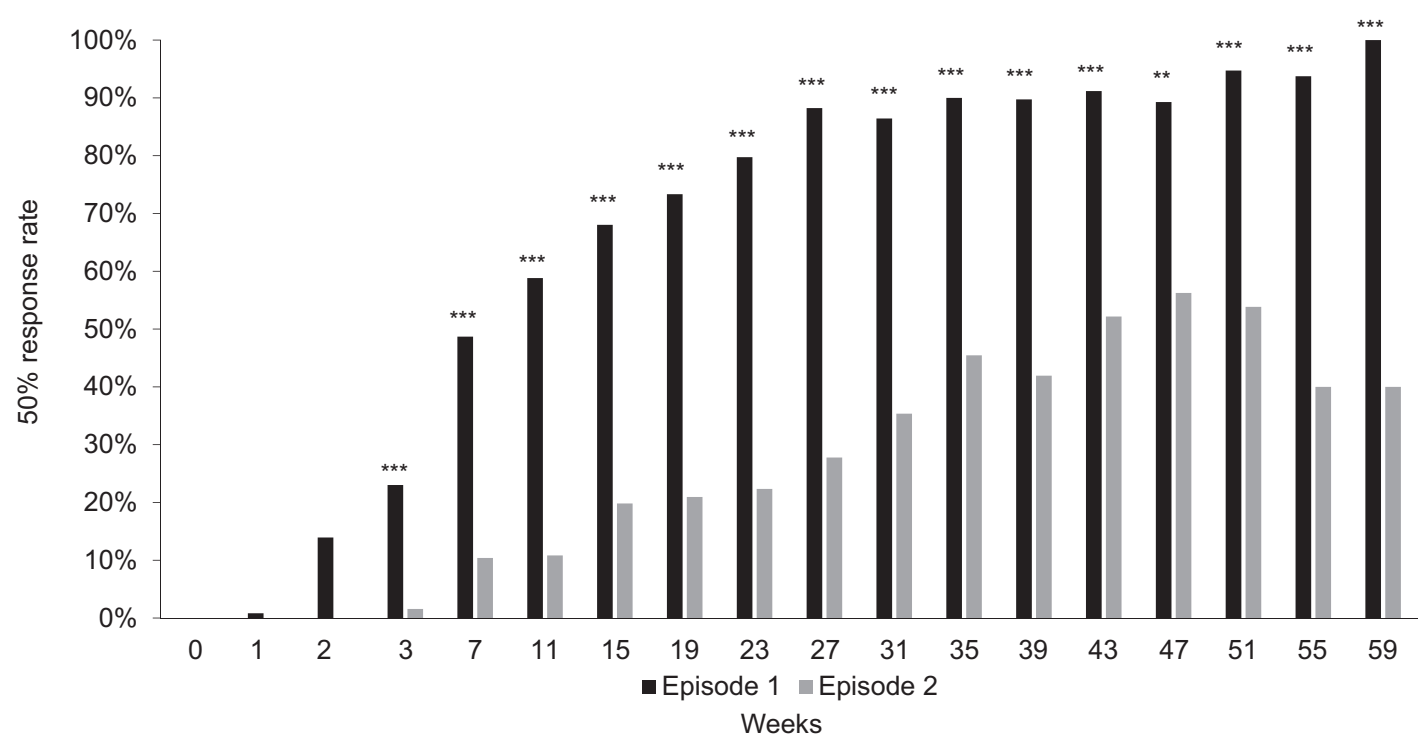


Fig. 3 a Changes in $20 \%$ response rates over time in first vs. second episodes $(N=130)$. The generalized estimating equation analysis revealed a significant interaction between episode and time $(F=75.2, P<0.001)$. ${ }^{* *} P<0.001,{ }^{*} P<0.01$. b Changes in $50 \%$ response rates over time in first vs. second episodes $(N=130)$. The generalized estimating equation analysis revealed a significant interaction between episode and time $(F=105.0, P<0.001)$. ${ }^{* *} P<0.001, * * P<0.01$

clinical practice. Furthermore, a recent analysis demonstrated that $50 \%$ of patients who discontinued oral paliperidone, 1-monthly long-acting injectable paliperidone, or 3-monthly long-acting injectable paliperidone did not experience relapse for approximately 2 months, 6 months, and 13 months, respectively [31]. As such, long-acting injectable antipsychotics may warrant consideration even for patients stabilized on oral antipsychotics with good medication adherence. As noted, non-adherence is particularly problematic in first episode psychosis [4] and, at the same time, risk of relapse may be even more relevant in this population.

These findings are in line with the psychosis "neurotoxicity" hypothesis and evidence that duration of untreated psychosis is associated with changes in neurocognitive performance and brain structure [32]. Time may be less relevant than psychosis per se; here, for example, the duration between the first and second episodes was as short as 19.3 weeks. In addition, both time to response as well as degree of response are impacted. More specifically, it took longer to respond following a second episode and fewer individuals attained a $50 \%$ reduction in overall symptom severity (i.e., much improvement) compared to a $20 \%$ reduction (i.e., minimal improvement) or positive symptom remission. Importantly, here we are looking at only 1 relapse and it is plausible that response is further attenuated with repeated relapses.

Limitations to the current investigation should be noted. First and foremost is the lack of blinding, although arguably this can also be seen as a strength because the data reflect "real world" clinical practice. Second, there is no absolute agreement of what constitutes "clinical response" or "clinical relapse", although various outcomes of antipsychotic treatment response were examined. Third, although numerous strategies were implemented to encourage and monitor adherence, pill counts were used instead of electronic monitoring to measure non-adherence to medication, which may have underappreciated non-adherence. Forth, some clinically important information (e.g., duration of untreated psychosis, antipsychotic dose at each visit, and reasons for non-adherence) was not collected on a regular basis or in a systematic fashion, or was missing. Fifth, the dataset primarily included patients who achieved positive symptom remission at the end of the first episode and experienced relapse because of non-adherence, which made it difficult to compare clinically important groups (e.g., remitters vs. non-remitters and patients who relapsed despite being adherent vs. those not being adherent). Critical to the conclusions drawn here is the fact that this was only the second psychotic episode, and it remains unclear as to whether the same results would be achieved across repeated episodes or if a longer gap occurred before re-initiation of treatment.

In conclusion, this is the first investigation to report response rates in a sample of individuals who experienced a first episode of psychosis, remitted in terms of positive symptoms with treatment, chose to discontinue pharmacotherapy and relapsed, leading to reinstatement of the same antipsychotic. The evidence here suggests that for most of these individuals it is possible to reestablish response, at least following 1 episode of relapse; this said, the response may be attenuated and/or delayed. From a clinical practice standpoint, this represents important information that must be incorporated into discussions regarding antipsychotic discontinuation. In short, the message to be conveyed is that good response cannot be guaranteed in the face of a relapse, even if the same treatment is reinitiated.

\section{FUNDING AND DISCLOSURE}

H.T. has received fellowship grants from the Canadian Institutes of Health Research (CIHR), Centre for Addiction and Mental Health (CAMH) Foundation, the Japanese Society of Clinical Neuropsychopharmacology, and Astellas Foundation for Research on Metabolic Disorders, speaker's honoraria from Meiji-Seika Pharma, Mochida, Otsuka, Sumitomo Dainippon Pharma, and Yoshitomi Yakuhin, and manuscript fees from Sumitomo Dainippon Pharma. C.S. has received funding and consulting fees and research support from Sunovion, Pfizer, and Dainippon Sumitomo Pharma, Centre for Addiction and Mental Health (CAMH), Hong Kong Health and Medical Research Grant, and the Chinese University of Hong Kong. G.R. has received research support from Novartis and HLS. G.Fervaha has no competing interests to disclose. G.Foussias has served on advisory boards for Hoffman-La Roche and Takeda, and received speaker's fees from Hoffman-La Roche, Lundbeck, and Novartis. R.B.Z. has received speaker's honoraria and an educational grant from Janssen-Ortho (Johnson \& Johnson). O.A. has received speaker's honoraria from Janssen-Ortho (Johnson \& Johnson), Lundbeck, Mylan Pharmaceuticals, HLS, and Novartis, and consultant fees from Janssen-Ortho (Johnson \& Johnson), Lundbeck, Otsuka, and Sumitomo Dainippon Pharma, and research support from Boehringer Ingelheim, Neurocrine Biosciences, Janssen-Ortho (Johnson \& Johnson), Otsuka, and Sunovion.

\section{ADDITIONAL INFORMATION}

Supplementary Information accompanies this paper at (https://doi.org/10.1038/ s41386-018-0278-3).

Publisher's note: Springer Nature remains neutral with regard to jurisdictional claims in published maps and institutional affiliations.

\section{REFERENCES}

1. Pennington $M, M c C r o n e ~ P$. The cost of relapse in schizophrenia. Pharmacoeconomics. 2017;35:921-36.

2. Leucht S, Tardy M, Komossa K, Heres S, Kissling W, Salanti G, et al. Antipsychotic drugs versus placebo for relapse prevention in schizophrenia: a systematic review and meta-analysis. Lancet. 2012;379:2063-71.

3. Sendt K-V, Tracy DK, Bhattacharyya S. A systematic review of factors influencing adherence to antipsychotic medication in schizophrenia-spectrum disorders. Psychiatry Res. 2015;225:14-30.

4. Mojtabai R, Lavelle J, Gibson PJ, Sohler NL, Craig TJ, Carlson GA, et al. Gaps in use of antipsychotics after discharge by first-admission patients with schizophrenia, 1989 to 1996. Psychiatr Serv. 2002;53:337-9.

5. Higashi K, Medic G, Littlewood KJ, Diez T, Granström O, Hert M, De. Medication adherence in schizophrenia: factors influencing adherence and consequences of nonadherence, a systematic literature review. Ther Adv Psychopharmacol. 2013;3:200-18.

6. Leucht S, Leucht C, Huhn M, Chaimani A, Mavridis D, Helfer B, et al. Sixty years of placebo-controlled antipsychotic drug trials in acute schizophrenia: systematic review, bayesian meta-analysis, and meta-regression of efficacy predictors. Am J Psychiatry. 2017;174:927-42.

7. Zhu Y, Li C, Huhn M, Rothe P, Krause M, Bighelli I, et al. How well do patients with a first episode of schizophrenia respond to antipsychotics: a systematic review and meta-analysis. Eur Neuropsychopharmacol. 2017;27:835-44.

8. Keating D, McWilliams S, Schneider I, Hynes C, Cousins G, Strawbridge J, et al. Pharmacological guidelines for schizophrenia: a systematic review and comparison of recommendations for the first episode. BMJ Open. 2017;7:e013881.

9. Wiersma D, Nienhuis FJ, Slooff CJ, Giel R. Natural course of schizophrenic disorders: a 15-year followup of a Dutch incidence cohort. Schizophr Bull. 1998:24:75-85. 
10. Emsley R, Chiliza B, Asmal L. The evidence for illness progression after relapse in schizophrenia. Schizophr Res. 2013a;148:117-21.

11. Lieberman J, Alvir JM, Koreen A, Geisler S, Chakos M, Sheitman B, et al. Psychobiologic correlates of treatment response in schizophrenia. Neuropsychopharmacology. 1996;14:13S-21S.

12. Emsley R, Oosthuizen P, Koen L, Niehaus D, Martinez L. Comparison of treatment response in second-episode versus first-episode schizophrenia. J Clin Psychopharmacol. 2013b;33:80-3.

13. Emsley R, Nuamah I, Hough D, Gopal S. Treatment response after relapse in a placebo-controlled maintenance trial in schizophrenia. Schizophr Res. 2012;138:29-34

14. American Psychiatric Association. DSM-IV-TR. Diagnostic Stat Man Ment Disord 4th Ed TR. 2000

15. Guy W. ECDEU Assessment Manual for Psychopharmacology. US Dep Heal Educ Welf Publ ADM 76-338. Rockville, MD: National Institute of Mental Health; 1976.

16. Overall JE, Gorham DR. The brief psychiatric rating scale. Psychol Rep. 1962;10:799-812.

17. Leucht S, Kissling W, Davis JM. The PANSS should be rescaled. Schizophr Bull. 2010;36:461-2.

18. Obermeier M, Mayr A, Schennach-Wolff R, Seemüller F, Möller H-J, Riedel M. Should the PANSS be rescaled? Schizophr Bull. 2010;36:455-60.

19. Leucht S, Kane JM, Kissling W, Hamann J, Etschel E, Engel RR. What does the PANSS mean? Schizophr Res. 2005;79:231-8.

20. Leucht S, Samara M, Heres S, Patel MX, Woods SW, Davis JM. Dose equivalents for second-generation antipsychotics: the minimum effective dose method. Schizophr Bull. 2014;40:314-26.

21. Lally J, Ajnakina O, Di Forti M, Trotta A, Demjaha A, Kolliakou A, et al. Two distinct patterns of treatment resistance: clinical predictors of treatment resistance in first-episode schizophrenia spectrum psychoses. Psychol Med. 2016;46:3231-40.

22. Demjaha A, Lappin JM, Stahl D, Patel MX, MacCabe JH, Howes OD, et al. Antipsychotic treatment resistance in first-episode psychosis: prevalence, subtypes and predictors. Psychol Med. 2017;47:1981-9.
23. Mena C, Gonzalez-Valderrama A, Iruretagoyena B, Undurraga J, Crossley NA. Early treatment resistance in a Latin-American cohort of patients with schizophrenia. Schizophr Res. 2018;199:380-5.

24. Howes OD, McCutcheon R, Agid O, Bartolomeis A, de, Beveren NJM, van, Birnbaum ML, et al. Treatment-resistant schizophrenia: Treatment Response and Resistance in Psychosis (TRRIP) Working Group Consensus Guidelines on Diagnosis and Terminology. Am J Psychiatry. 2017;174:216-29.

25. Goff DC, Falkai P, Fleischhacker WW, Girgis RR, Kahn RM, Uchida H, et al. The longterm effects of antipsychotic medication on clinical course in schizophrenia. Am J Psychiatry. 2017;174:840-9.

26. Correll CU, Rubio JM, Kane JM. What is the risk-benefit ratio of long-term antipsychotic treatment in people with schizophrenia? World Psychiatry. 2018;17:149-60.

27. Kishimoto $T$, Robenzadeh A, Leucht $C$, Leucht $S$, Watanabe $K$, Mimura $M$, et al. Long-acting injectable vs oral antipsychotics for relapse prevention in schizophrenia: a meta-analysis of randomized. Trials . 2014;40:192-213.

28. Ostuzzi G, Bighelli I, So R, Furukawa TA, Barbui C. Does formulation matter? A systematic review and meta-analysis of oral versus long-acting antipsychotic studies. Schizophr Res. 2017;183:10-21.

29. Kishimoto T, Nitta M, Borenstein M, Kane JM, Correll CU. Long-acting injectable versus oral antipsychotics in schizophrenia: a systematic review and metaanalysis of mirror-image studies. J Clin Psychiatry. 2013;74:957-65.

30. Kishimoto T, Hagi K, Nitta M, Leucht S, Olfson M, Kane JM, et al. Effectiveness of long-acting injectable vs oral antipsychotics in patients with schizophrenia: a meta-analysis of prospective and retrospective cohort studies. Schizophr Bull. 2018:44:603-19.

31. Weiden PJ, Kim E, Bermak J, Turkoz I, Gopal S, Berwaerts J. Does half-life matter after antipsychotic discontinuation? A relapse comparison in schizophrenia with 3 different formulations of paliperidone. J Clin Psychiatry. 2017;78: e813-20.

32. Rund BR. Does active psychosis cause neurobiological pathology? A critical review of the neurotoxicity hypothesis. Psychol Med. 2014;44:1577-90. 